\title{
Análisis de las competencias y roles del Instituto del Niño Adolescente del Uruguay
}

Analysis of the competences and roles of the Institute for Children and Adolescents of Uruguay

Análise das competências e funções do Instituto da Criança e do Adolescente do Uruguai

José Luis Priore Di Salvatore ${ }^{1}$

\section{Resumen}

El estudio exploró y analizó el desempeño de los niveles de meso y micro gestión del Instituto del Niño y Adolescente del Uruguay (INAU) de acuerdo a los roles y funciones atribuidas en el marco de Política Pública (PP). El diseño metodológico fue de carácter exploratorio descriptivo y analítico, mediante estudio de casos múltiple, utilizando metodología cuali y cuantitativa. Se identificaron las áreas de micro y mesogestión institucional como las conformadas por las 19 Direcciones Departamentales y las Subdirecciones Generales Territorial y Programática respectivamente. Mediante encuestas y entrevistas en profundidad se describieron y analizaron los niveles de desempeño en el rol asignado a través de la legislación vigente. También se realizó un grupo focal con agencias de infancia externas al instituto y revisión documental para completar los objetivos del estudio. Los resultados mostraron que la institución se encuentra en un proceso de readecuación caracterizado por un cambio de modelo de gestión en el cual existen líneas estratégicas comunes desde el nivel central de mesogestión hacia el territorio. Estas líneas institucionales mostraron buenos grados de desempeño a nivel de la microgestión, principalmente en el área de ejecución que también responde al gran nivel de demanda de atención existente y en el que el instituto parece ser el único con capacidad de respuesta. Esto tiene consecuencias en las otras dos áreas de gestión: planificación y principalmente la supervisión. En referencia a este último rol, la supervisión se aplica principalmente en agencias contratadas por el instituto, y tiene poca incidencia a nivel general de la política pública de infancia del país. Esto también podría ser consecuencia del nivel subordinado que ocupa el instituto en el escenario nacional de política de infancia. Sin embargo, existen experiencias de

${ }^{1}$ Licenciado en Enfermería. Universidad de la República. Email: jpriore@adinet.com.uy ORCID: 0000-0002-7537-2269 
participación del instituto en espacios interinstitucionales en los que se evalúa satisfactoriamente la función supervisora.

\section{Palabras clave:}

Política pública, organización y administración, defensa del niño.

\section{Abstract}

This study explored and analyzed the performance of the meso and micro management levels of the Institute for Children and Adolescents of Uruguay (INAU) according to the roles and functions assigned with a Public Policy approach. The methodological design was exploratory, descriptive and analytical, through a multiple case study, using qualitative and quantitative methodology. The institutional micro and meso-management areas were identified as those made up of the 19 Departmental Directorates and the Territorial and Programmatic General Subdirectorates respectively. Through surveys and in-depth interviews, the performances in the role assigned through the current legislation were described and analyzed. A focus group was also held with children's agencies outside the institute and a documentary review to complete the objectives of the study. The results showed that the institution is in a readjustment process characterized by a change in the management model in which there are common strategic lines from the central meso-management level to the territory. These institutional lines showed good levels of performance at the level of micromanagement, mainly in the execution area, which also responds to the high level of demand for existing care and in which the institute seems to be the only one with the capacity to respond. This has consequences for the other two management areas: planning and mainly supervision. This last role, supervision, is applied mainly in the agencies contracted by the institute, and has little impact at the general level of public policy on childhood in the country. This could also be a consequence of the subordinate level that the institute occupies in the national scenario of childhood policy. However, there are experiences of the institute's participation in inter-institutional spaces in which the supervisory function is satisfactorily evaluated.

\section{Keywords:}

Public Policy, Organization and Administration, Defense of the Child.

\section{Resumo}

Este estudo explorou e analisou o desempenho dos níveis de meso e microgestão do Instituto da Criança e do Adolescente do Uruguai (INAU) de acordo com os papéis e funções atribuídos com um enfoque das Políticas Públicas. O desenho metodológico foi exploratório, descritivo e analítico, por meio de um estudo de caso múltiplo, com metodologia qualitativa e quantitativa. As áreas de micro e meso-gestão foram identificadas como aquelas compostas pelas 19 Direcções Departa- 
mentais e pelas Subdirectorias Gerais Territorial e Programática, respectivamente. Por meio de pesquisas e entrevistas em profundidade, foram descritos e analisados os níveis de desempenho nas funções atribuídas pela legislação em vigor. Um grupo focal também foi realizado com agências infantis fora do instituto e uma revisão documental para completar os objetivos do estudo. Os resultados mostraram que a instituição está em um processo de readequação caracterizado por uma mudança no modelo de gestão em que existem linhas estratégicas comuns desde a meso-gestão central até o território. Estas linhas institucionais apresentaram bons niveis de desempenho ao nível da microgestão, principalmente na área de execução, o que também responde ao elevado nível de procura de cuidados existentes e em que o instituto parece ser o único com capacidade para responder. Isso tem consequências para as outras duas áreas de gestão: planejamento e principalmente supervisão. No que se refere a esta última função, a fiscalização é aplicada principalmente nas agências contratadas pelo instituto e tem pouco impacto no nível geral das políticas públicas infantis do país. Isso também pode ser consequência do nível subalterno que o instituto ocupa no cenário nacional da política da infância. No entanto, existem experiências de participação do instituto em espaços interinstitucionais em que a função de fiscalização é avaliada de forma satisfatória.

\section{Palavras-chave:}

Política pública, organização e administração, defesa da criança.

\section{Introducción}

Parece existir acuerdo respecto que la infancia es un fenómeno cultural que ha dependido del contexto socio histórico y que ha tenido diferentes visiones a lo largo de la historia. Aries Ariès (1987) plantea la existencia de tres tipos de infancia: un primer tipo en la que se visualiza un infante desprotegido y a la vez adoctrinado a la adultez, un segundo tipo que surge del producto de la innovación tecnológica en la que los niños y adolescentes se introducen al mundo adulto y un tercer tipo en la que identifica una infancia desprotegida y que es considerado por la sociedad y la pedagogía un problema y un peligro. Estos niños son así derivado a manos del Estado y donde para entender a estos "menores" ya no se acude a tratados pedagógicos sino principalmente a manuales penales o psiquiátricos, cambiando su lugar de tránsito evolutivo desde la escuela a un instituto correccional o la cárcel. Es de esta forma que entra en juego el rol del Estado para asumir el cuidado de esta infancia desprotegida. La evolución de la normativa en nuestro país para niños, niñas y adolescentes (NNA), que siempre giró en los ejes familia y Estado, comenzó a consolidar su matriz tutelar sobre inicios del SXX y se consolidó luego del transcurso del tiempo en el INAU, pero con un espíritu de ser el instituto que liderara la PP de toda la infancia y no solamente la de los menores de edad en situación de riesgo. De esta forma, el Estado a través de diferentes instituciones a lo largo del tiempo ha asumido el lugar a cargo del cuidado de NNA y el INAU actualmente ocupa el además de ese cuidado el rol asignado por Ley de liderar la PP de infancia. Por tanto, tal como refieren diversos autores, ese rol de 
acción debe ser ejercido promoviendo determinadas actividades, produciendo determinados bienes, ofreciendo ciertos servicios o regulando determinadas formas de interacciones sociales, en una forma dinámica en la que los roles son múltiples y cambian permanentemente a los efectos de alcanzar un objetivo preestablecido y que en el caso del instituto están definidas por la legislación a través de la Ley 17.823.

Es así, que la ratificación de la Convención de los derechos del Niño (CDN) en los países ha traído aparejada la necesidad de incorporar el mandato de protección integral de niños, niñas y adolescentes NNA a la política pública de las naciones. Surgen así las legislaciones que designan organismos y encomiendan de diferentes formas y estrategias la gestión sobre niñez y familia en los Estados que han adherido al cumplimiento del acuerdo. El INAU cuenta con un documento denominado Manual de Estructuras y Funciones (2018) es un documento que aprueba la estructura orgánica del Instituto y donde se describen y establecen las funciones específicas, dependencias y coordinación de las distintas unidades organizacionales. En él encontramos que, en concordancia con lo establecido en el cumplimiento del Art. 68 de la Ley 17.823 se describe al INAU como el organismo encargado de la función de programar y ser rector de las políticas de niñas, niños y adolescentes, pero también le asigna la ejecución de las mismas en diferentes modalidades de atención, propias o coordinadas con otras instituciones públicas y privadas y con la sociedad civil. $\mathrm{Al}$ interiorizarnos en el mencionado manual, encontramos la descripción de las Unidades Organizativas que integran las áreas de la microgestión institucional comprendidas y organizadas en las Direcciones Departamentales (DD), con sus relaciones jerárquicas, grados de dependencia, objetivos, naturaleza de la función y las actividades clave más relevantes o vinculantes al área de consideración. Por otra parte se identificaron en el documento dos unidades organizacionales de la institución que a los efectos del estudio se consideraron que ocupaban el rol de mesogestión denominadas Sub Dirección General Programática (SDGP) y la Sub Dirección General de Gestión Territorial (SDGT). En referencia a estas dos áreas de gestión se las mismas fueron identificadas de acuerdo al documento y vinculadas con el rol planificador-rector- fiscalizador $y$ al rol ejecutor respectivamente. También se identificaron las competencias signadas en el nivel de microgestión de las DD y que junto a las áreas meso se indagaron y sometieron a análisis. Dado que toda institución requiere de un área administrativa para el cumplimiento de sus fines, la Sub Dirección General Administrativa, que jerárquicamente se encuentra en la organización a nivel meso no fue considerada en la presente investigación. Tampoco se consideraron otras áreas de Monitoreo institucional y Planificación Estratégica, dado que la investigación indagó hacer foco en las líneas de interacción entre las dos Sub Direcciones (nivel meso) y las DD (nivel micro).

Por otra parte, la investigación buscó profundizar el conocimiento acerca del lugar jerárquico que el Instituto ocupa a nivel de las PP de NNA y en su relación con el Ministerio de desarrollo Social (MIDES), además de describir fortalezas y dificultades para poder operacionalizar determinadas actividades institucionales. El estudio se orientó hacia los aspectos de gestión de corto, mediano y largo plazo en las áreas programática y de rectoría, como consecuencia de la necesidad del cumplimiento simultáneo en 


\section{Objetivo Principal}

territorio de los aspectos de ejecución. De esta forma y tal como se plantea en la hipótesis, se podría pensar que el rol ejecutor de las políticas de infancia a nivel territorial implicaría un gran esfuerzo en la gestión institucional y que ello podría tener consecuencias en el rol programático-fiscalizador de dicha política, como plantean diversos autores: "Básicamente, se señala que el INAU ha reemplazado el antiguo Instituto Nacional del Menor y sus funciones clásicas, que representaba el sistema tutelar en el Uruguay. Sin embargo, para muchos autores la matriz de la institucionalidad no ha cambiado substancialmente y la transformación institucional y de prácticas ha sido muy lenta. En este sentido, daría la impresión que gran parte de los esfuerzos del INAU están en el ámbito de la ejecución y cumplimiento de la multiplicidad de funciones que le da el Código y no en la necesaria coordinación y en la formulación de políticas sociales para toda la infancia y la adolescencia" (Morlachetti, 2013, p.68) "Lo antes expuesto explicaría en parte las razones por las que se «camina lento» en la implementación de las políticas; existen decisiones tomadas con presupuestos aprobados y gran parte de la población valora y demanda las acciones...sin embargo múltiples factores implicados en la gestión hacen que los procesos se enlentezcan y los plazos se alarguen más allá de los previsto. Mientras tanto el tiempo pasa y las generaciones también y aunque en el nivel discursivo se proponga lo contrario, una parte de la población permanece aún "librada a su suerte". (Cerutti, Canetti, Duarte, Parafita, 2014, p.44)
Generar conocimiento sobre el desempeño de los niveles de meso y micro gestión del INAU y analizar los mismos en referencia a los roles y funciones atribuidas.

\section{Objetivos específicos}

- Describir los principales preceptos institucionales a nivel de mesogestión del Instituto y su aplicación en relación a las acciones que desempeñan el nivel de microgestión.

- Conocer las principales características del desempeño de cada uno de los niveles en lo que refiere a su composición, objetivos, funcionamiento, actividades y resultados.

- Indagar acerca de la estructura de las diferentes áreas de microgestión, y cómo se desempeñan los roles asignados en territorio, analizando potencialidades, limitaciones $y$ conflictos.

\section{Hipótesis de Partida}

Los preceptos institucionales y lineamientos estratégicos del INAU definidos por las áreas de mesogestión son eficaces cuando logran una aplicación plena a nivel de la microgestión en el rol ejecutor-territorial, pero también en los roles programático y fiscalizador.

\section{Diseño Metodológico}

Se planteó un diseño metodológico de carácter exploratorio descriptivo y posterior análisis acerca del funcionamiento de los diferentes niveles de meso y microgestión. El diseño se reali- 
zó mediante estudio de casos múltiple, utilizando variadas fuentes de evidencias e información y armonizando procedimientos cuantitativos y cualitativos, y cuya meta es construir una teoría diferente para explicar y aclarar determinado fenómeno (Yin, 1994, Neiman y Quaranta, 2006). Por tanto, la investigación permitió obtener resultados a través de situaciones en la cual hay muchas más variables de interés que datos observacionales, basándose en variadas fuentes de evidencias y favoreciéndose de esta manera el proceso previo de propuestas teóricas que tutelan la recolección y el análisis de datos. (Monge, 2010). esta metodología combinada intentó, tal como lo expresan Teddliey Tashakkori (2003), armonizar diseños metodológicos en base a variadas fuentes de evidencia a fin de lograr no sólo un mejor conocimiento del objeto de investigación, sino que ir más allá y realizar una triangulación o convergencia de los resultados obtenidos por diferentes metodologías, buscando verificar si los diferentes métodos y fuentes ofrecen los mismos resultados, probando así la robustez de los mismos en explicar y analizar la realidad. Por tanto, al indagar en dichos cometidos institucionales y niveles de desempeño acerca de los roles y funciones atribuidas, era necesario contar con objetivos que se consideran imprescindibles alcanzar, y que son asumidos por la institución cuya finalidad es dirigir las acciones para lograr corregir una situación observada como insatisfactoria o inconveniente.

Para el área de microgestión se construyeron un total de 25 indicadores que aplicaron a las 19 DD a saber: 11 indicadores de ESTRUCTURA (3 en relación a los proyectos existentes, 4 en relación a los $\mathrm{RRHH}$ existentes y 4 en relación a las organizaciones contratadas); 3 de PROCE-
SO (que midieron la extensión de utilización de los servicios hacia NNA); 2 de RESULTADO (en relación al desempeño de las DD) y 9 indicadores de CALIDAD relacionados a las áreas de planificación, ejecución y rectoría. Para el área de mesogestión se realizaron dos entrevistas individuales en profundidad a representantes de las dos Sub Direcciones mencionadas. En tercer lugar se realizó una reunión de grupo focal con representantes de instituciones referentes en NNA; finalmente se acudió además de revisión documental. Todos los datos fueron recabados exclusivamente por el autor y procesados en forma confidencial. A nivel de microgestión se procedió al cálculo de los indicadores de estructura, proceso y resultado. Las variables cualitativas se categorizaron para favorecer el análisis y a los efectos de procesar los datos poblacionales de NNA se realizó una prueba de validez de la distribución normal de la población. Para ello se estableció como hipótesis nula la normal distribución de NNA atendidos y como hipótesis alternativa que los índices no presentaban una distribución normal. Seguidamente se aplicó la Prueba de Shapiro -Wilk con los índices de NNA atendidos de los 19 departamentos buscando contrastar la normalidad del conjunto de datos. Se calculó la media y el DE y se utilizó como índice de confianza un valor de 95 \% con una significancia de error del $5 \%$. Además, se procedió a investigar la existencia de relación entre variables mediante pruebas no paramétricas. Mediante tablas de correlación se buscó asociación entre las variables de estructura y proceso con las de resultado y calidad. Para ello, se procedió a categorizar todos los datos de las encuestas en una base para el posterior análisis mediante el cálculo de indicadores y análisis de correlaciones bivariadas. Para verificar la con- 
cordancia, dirección y valor la fuerza de la relación entre las variables se utilizaron las pruebas Rho de Sperman y Tau-b de Kendall según se utilizaran variables continuas o discretas en el respectivo cálculo. El nivel de significación se estableció para todos los casos como menor de 0.05. Los análisis estadísticos se realizaron con el programa: Statistical Package for the Social Sciences (SPSS.V.22). A nivel meso los datos obtenidos de las entrevistas en profundidad fueron anonimizados y analizados mediante las desgrabaciones correspondientes con las autorizaciones de los entrevistados. Igual proceso se desarrolló con las grabaciones de la reunión de grupo focal. Los datos correspondientes a documentos consultados provinieron de información pública disponible, principalmente de la Plataforma Integrada de Transparencia y Análisis para la Gestión de la Administración de INAU. La investigación contó con la autorización del Directorio del INAU (Resol 61955/2019 MS/sp) y aval del Comité de Ética de la Facultad de Psicología, ajustándose a los requerimientos normativos establecidos en la Ley 18331 y el Decreto No 158/019. Los gastos derivados de la investigación fueron costeados personalmente por el investigador dado que el estudio no contó con fuentes de financiación.

\section{Consideraciones Éticas y autorizaciones}

\section{institucionales}

Para la realización de la presente investigación, se cursó nota de solicitud de autorización al Directorio del INAU la que generó el expediente $60022 / 19$ que fue tratado y autorizado en sesión de Directorio del 18 de diciembre de 2019 y Resolución 61955/2019 MS/sp del 27 de diciembre de 2019. El aval fue enviado desde INAU me- diante correo electrónico institucional a conocimiento de las19 DD y ambas Sub Direcciones Generales.

Por otra parte contó con el aval del Comité de Ética de la Investigación de Facultad de Psicología a los efectos de ajustarse a los requerimientos normativos establecidos en la Ley 18331 y el Decreto N. ${ }^{\circ}$ 158/019 del MS del año 2019, habiendo sido autorizado por el mencionado Comité con fecha 3 de marzo de 2020.

\section{Resultados y Análisis}

A continuación se exponen los hallazgos más relevantes:

Según los datos relevados, casi el 11\% del total de NNA del país se encuentran vinculados a algún tipo de proyecto de INAU en un porcentaje que es bastante similar y con una distribución independientemente del número de habitantes de cada departamento, de acuerdo a la prueba de validez de la distribución normal de la población. El índice medio de atendidos calculado para los 19 departamentos fue de $0.1238 \mathrm{DE}$ (0.029). El valor estadístico hallado mediante la prueba de Shapiro-Wilk para los índices de distribución de NNA atendidos por INAU en los 19 departamentos mostró un valor $\mathrm{p}=0.642$ (no significativo).

De todos los NNA vinculados a INAU, casi el $90 \%$ se encuentran mediante proyectos de instituciones contratadas por la institución (Tabla 1) y el resto atendido por la propia institución. En referencia al número de proyectos por NNA (primer indicador de estructura), el promedio es de 2.65 proyectos por NNA atendido de los cuales $10.56 \%$ corresponden a proyectos pro- 
pios de INAU y un $89.44 \%$ a proyectos de instituciones externas que trabajan para INAU. Este valor representa un promedio de 0.27 proyectos por NNA a cargo de INAU y 2.37 proyectos por NNA a cargo de organizaciones contratadas con una relación entre instituciones contratadas e INAU de 6 a 1 respectivamente (Tabla 2 )

Tabla 1.

Distribución de NNA por Departamento e indice de atención

\begin{tabular}{lccc}
\hline Departamento & Núm. total NNA & Núm. NNA atendidos por & Índice de NNA atendidos \\
\cline { 2 - 4 } & del Depto. & INAU del Depto. & \\
\hline Artigas & 23717 & 13083 & 0.1301 \\
\hline Canelones & 144797 & 4184 & 0.0904 \\
\hline Cerro Largo & 25803 & 3035 & 0.1622 \\
\hline Colonia & 32004 & 2928 & 0.0948 \\
\hline Durazno & 17318 & 1330 & 0.1691 \\
\hline Flores & 6849 & 2351 & 0.1942 \\
\hline Florida & 18652 & 1532 & 0.1260 \\
\hline Lavalleja & 15556 & 3274 & 0.0985 \\
\hline Maldonado & 46003 & 30884 & 0.0712 \\
\hline Montevideo & 308451 & 5004 & 0.1001 \\
\hline Paysandú & 34518 & 2093 & 0.1450 \\
\hline Rio Negro & 17317 & 3980 & 0.1209 \\
\hline Rivera & 32407 & 2683 & 0.1228 \\
\hline Rocha & 18709 & 4612 & 0.1434 \\
\hline Salto & 39819 & 3433 & 0.1158 \\
\hline San José & 29568 & 2926 & 0.1161 \\
\hline Soriano & 24058 & 2878 & 0.1216 \\
\hline Tacuarembó & 27383 & 1772 & 0.1051 \\
\hline Treinta y Tres & 14092 & & 0.1257 \\
\hline & & & \\
\hline & & & \\
\hline
\end{tabular}

Fuente: Plataforma Integrada de Transparencia y Análisis para la Gestión de la Administración de INAU Elaboración propia.

El segundo componente de estructura que hace referencia a la proporción de recursos humanos propios de la institución y su distribución en las tres áreas de estudio (planificación, atención y supervisión o rectoría) mostraron un valor promedio de 0.44 funcionarios por NNA. Con respecto a la distribución de los mismos, si bien el 100 \% de las DD manifestaron tener RRHH destinados a cada una de las áreas de gestión, también se indagó la exclusividad, teniendo en cuenta que la misma representa una fortaleza en el desempeño de cada área. Los datos obtenidos mostraron que las áreas de gestión son compartidas por muchos de ellos y existen funcionarios que se desempeñan en más de un área. Al relevar la dedicación exclusiva a cada uno de los componentes se encuentra 
Tabla 2.

Relación entre proyectos y NNA.

\begin{tabular}{|c|c|c|c|c|}
\hline Componente / Área & Descripción & Indicador/es & Cálculo & $\underline{\text { Valor }}$ \\
\hline \multirow[t]{3}{*}{$\begin{array}{l}\text { ESTRUCTURA } \\
\text { Relación entre los } \\
\text { proyectos existentes } \\
\text { en la DD y NNA }\end{array}$} & $\begin{array}{c}\text { Medir la } \\
\text { relación entre } \\
\text { los proyectos } \\
\text { disponibles y } \\
\text { cantidad de } \\
\text { NNA a la que } \\
\text { está destinadas } \\
\text { las DD }\end{array}$ & $\begin{array}{l}\text { Promedio de proyectos totales de la DD en un } \\
\text { periodo X } 100 \\
\text { Promedio de NNA vinculados en ese periodo }\end{array}$ & $\begin{array}{c}132.63 \times 100 \\
5003\end{array}$ & 2,65 Proyectos/NNA \\
\hline & & $\begin{array}{l}\text { Promedio de proyectos propios de INAU } \\
\text { vinculados a la DD en un periodo X } 100 \\
\text { Promedio de NNA vinculados en ese periodo }\end{array}$ & $\begin{array}{c}14 \times 100 \\
5003\end{array}$ & $\begin{array}{c}0.27 \text { Proyectos/NNA } \\
(10.56 \%)\end{array}$ \\
\hline & & $\begin{array}{l}\text { Promedio de proyectos propios de ONG } \\
\text { vinculados a la DD en un periodo X } 100 \\
\text { Promedio de NNA vinculados en ese periodo }\end{array}$ & $\begin{array}{c}118.63 \times 100 \\
5003\end{array}$ & $\begin{array}{c}2.37 \text { Proyectos/NNA } \\
(89.44 \%)\end{array}$ \\
\hline $\begin{array}{l}\text { Fuente: Plataforma } \\
\text { Elaboración propia }\end{array}$ & Integrada de Tra & sparencia y Análisis para la Gestión de la Adm & ión de INAU & \\
\hline
\end{tabular}

que en el área de planificación no existen RRHH exclusivos, mientras que 17 DD manifestaron poseer RRHH exclusivos para atención (89.47 \%) y 2 DD manifestaron tener RRHH exclusivos para supervisión o rectoría en el Departamento. En este punto además, el 100\% de las DD reportaron que poseen RRHH destinados a planificación y atención, pero no así a rectoría (10.53\% no poseen). $\mathrm{Al}$ indagar sobre la especificidad en el área, la planificación no reporta RRHH específicos dado que la gestión del cuidado retiene a casi todos los RRHH vinculados a ella $(89.47 \%$ de especificidad). En supervisión también decae bastante la especificidad a $31.57 \%$. Casi todas la DD reportaron que es casi imposible destinar RRHH dedicados a la atención a que realicen otras actividades por la gran demanda que requiere, no pudiendo tener exclusividad.
El tercer componente de estructura abordó la participación de las instituciones contratadas por INAU en los tres componentes de gestión mencionados. A través de los datos reportados por las 19 DD un $63.15 \%$ manifiestan que las instituciones contratadas participan en actividades de planificación; el 100 \% reportan la participación en los procesos de atención y cuidado mientras que ninguna reporta participación en aspectos de rectoría.

Con respecto al componente de proceso, se indagó acerca de la extensión de la utilización del servicio que desarrolla la institución a través de los proyectos de atención propios y con organizaciones contratadas, buscando medir la proporción de población beneficiaria que hace uso de los mismos. Como se mencionó previa- 
mente, una media de $10.83 \%$ de los NNA del país se encuentra vinculado a algún proyecto o programa de la institución y se distribuyen en una importante proporción a favor de proyectos a cargo de instituciones contratadas en una relación de casi 6 a 1, con una proporción de 1.61 (que representa el $14.85 \%$ ) de vinculaciones propias de INAU y 9.22 (que representa el 85.15 \%) a cargo de instituciones contratadas.

El componente resultados midió el desempeño de las DD en términos de efectividad en las áreas de planificación y supervisión o rectoría, intentando describir cuanto se debilitaban estos aspectos debido a la importante asignación de RRHH al área de atención. A partir de los datos reportados por los integrantes de los equipos de dirección de las 19 DD se encontraron valores de cumplimiento que oscilaron desde un $45 \%$ hasta un $100 \%$, con un índice calculado promedio de $80.53 \%$ en el área de planificación y 81.58 $\%$ en el área de supervisión o rectoría.

El componente que correspondió al área de calidad referido y que estaba referido al desempeño de las actividades en las tres dimensiones estudiadas (planificación, atención y supervisión) se llevaron a cabo mediante el análisis de las percepciones de dichas funciones por parte de los participantes de la encuesta, categorizando el desempeño en muy bueno, bueno e insuficiente, y obteniéndose: a) en la dimensión de planificación la percepción reportada por 7 de las 19 DD fue catalogada como muy buena, (36.8\%) y las 12 restantes como buenas (63.2\%). Ninguna DD reportó la dimensión de planificación como insuficiente; b) en el área vinculada a la atención dicha función fue percibida como muy buena por 6 de los 19 integrantes de los equipos de Dirección, lo que representa el 31.6 $\%$; por otra parte $12 \mathrm{DD}$ las percibieron como buena, representando el $63.2 \%$ y una la cataloga como insuficiente (5.3\%); c) el componente de supervisión y rectoría, mostró que 6 de las 19 DD reportaron un desempeño como muy bueno (31.6 \%), 13 un desempeño bueno (68.4\%) y no hubo reportes de insuficiencia en el componente de dicha área. Los datos reportados en referencia a la percepción del nivel de efectividad en las áreas de planificación mostraron que los valores inferiores reportados fueron de $45 \%$ de y los más altos de $100 \%$. En la distribución de los mismos se observó que 15 DD (78.94 \%) concentran un nivel de efectividad en la gestión de la planificación en el entorno del 80 a $90 \%$.

Posteriormente, se aplicó para medir el desempeño en las áreas el coeficiente de correlación de Spearman. En relación al número de NNA por Departamento, se encontraron valores de asociación media para las tres áreas, pero no significativa (valores de $\mathrm{p}=0.075$ para planificación, $\mathrm{p}=0.074$ para atención y $\mathrm{p}=0.063$ para supervisión). En referencia a la relación entre el número de proyectos por NNA no se encontró asociación, así como tampoco en referencia al porcentaje de proyectos a cargo de instituciones externas. Todos los valores encontrados no fueron significativos y con coeficientes de asociación débil.

Además, se buscó también asociación que pudiera explicar los valores de desempeño en función a los RRHH existentes en lo que refiere al número de funcionarios de las DD y su relación al número de NNA del departamento. Los valores de correlación hallados fueron muy débiles y no significativos para las mencionadas va- 
riables (valores de $\mathrm{p}=0.568, \mathrm{p}=0.936$ y $\mathrm{p}=0.736$ para planificación, atención y supervisión respectivamente). Respecto al desempeño en las tres áreas vinculado al número de funcionarios de INAU por NNA también se encontró una asociación débil, con coeficientes de menos de 0.20 (aunque en las áreas de planificación y supervisión con valores negativos), pero también sin asociación significativa (valores de $\mathrm{p}=0.463$ para planificación, 0.557 para atención y 0.446 para supervisión).

En referencia a los resultados obtenidos de las entrevistas en profundidad se reportó que la institución se encuentra en un proceso de cambio de gestión aprobada por las autoridades y llevada a cabo desde la mesogestión hacia el territorio. Esto se está gestionando a través de la reorganización y trazado de líneas estratégicas desde el área de la SDGT confeccionado líneas estratégicas comunes al territorio y en el que estas líneas o mandatos institucionales contaron con la participación de las áreas de ejecución. De esta forma se pretende generar procesos de atención con un gran encuadre a nivel general del país mediante acciones en territorio que se encuentran enmarcadas en la estructura de las políticas sociales hacia NNA.

Este proceso de adecuación está instalando una estrategia territorial unificada, con mandatos institucionales comunes hacia el territorio, tendientes a generar procesos de atención unificados en el país. Estos lineamientos o preceptos institucionales son ejecutados en territorio en procesos de gestión que también responden a realidades particulares que se busca que respondan con cierta adaptabilidad local. Dentro de estas respuestas, si bien el proceso de reade- cuación y cambio de modelo de gestión intenta generar un sistema de protección más abierto y más centrado en el niño como sujeto de derecho, muchas veces se ejecutan fórmulas que son más de exclusión y que parecerían obedecer a la importante demanda de cuidado a la que es sometida la institución en territorio, principalmente en especialidades como salud mental y discapacidad, que no tienen término de finalización dado que estas personas permanecen en la institución más allá de la edad adulta.

Si bien las DD identifican las tres áreas de gestión en territorio (planificación, ejecución y rectoría), los recursos humanos están distribuidos en su mayoría en tareas de ejecución de cuidado en territorio en todas sus formas dedicando en promedio casi el 90\% de los RRHH (Tabla 3). Esto si bien es evaluado como muy positivo por la institución y organismos externos, en cierta medida ocurre por la gran demanda de cuidado que la situación representa y en la cual el INAU no cuenta con socios estatales para llevarlas a cabo, debiendo asumirlo solo. Este aspecto que podría tener consecuencias en la gestión de planificación y rectoría, no surgió de las encuestas de microgestión, en donde hubo coincidencia en los valores reportados en las tres áreas, en donde cerca del 70 \% de las DD cataloga la gestión como buena y casi la totalidad de las restantes como muy buena. (Tabla 4) Esto concuerda con los hallazgos del componente de calidad en la investigación cuantitativa en la que el porcentaje de cumplimiento en planificación y rectoría y el nivel de desempeño de las mismas, áreas tuvo asociación débil no significativa, pero que, respecto a la asociación entre desempeño exclusivo en planificación y rectoría, la investigación encontró asociación positiva y estadísticamente significativa, en ambas áreas. (Gráfico 1). 
Tabla 3.

Distribución de los RRHH Según Área de Gestión.

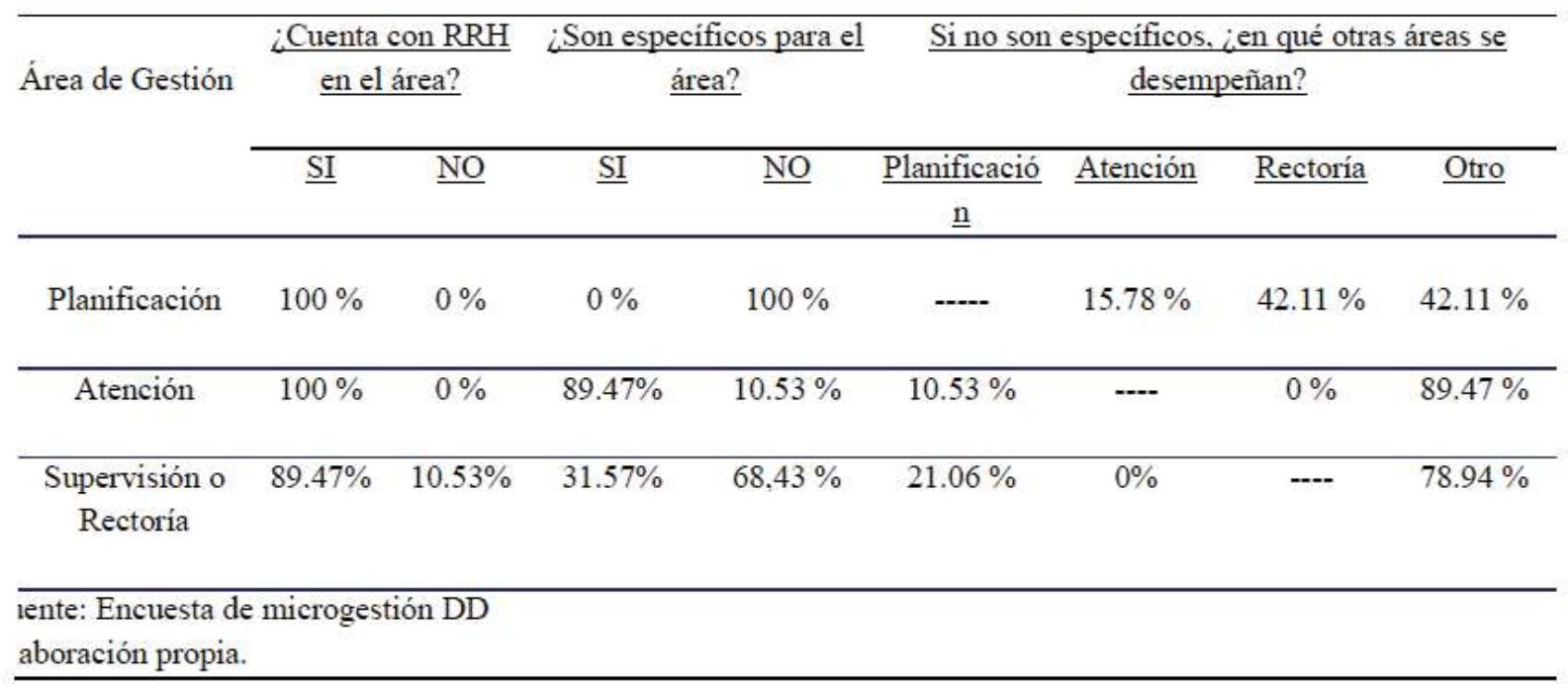

Tabla 4.

Desempeño Planificación, Atención y Supervisión de las DD

\begin{tabular}{lccc}
\hline & Muy bueno & $\underline{\text { Bueno }}$ & $\underline{\text { Insuficiente }}$ \\
\hline Planificación & $36,8 \%$ & $63,2 \%$ & $0 \%$ \\
\hline Atención & $31,6 \%$ & $63,2 \%$ & $5,3 \%$ \\
\hline Supervisión & $31,6 \%$ & $68,4 \%$ & $0 \%$ \\
\hline $\begin{array}{l}\text { Fuente: Encuesta de microgestión DD } \\
\text { Elaboración propia }\end{array}$ & & \\
\hline
\end{tabular}

Todas las DD manifestaron poseer RRHH destinados a la gestión de la planificación, (incluyendo un 63 \% que manifestó que las instituciones contratadas participan del proceso), pero ninguna cuenta con personal exclusivo y esa actividad es llevada a cabo por funcionarios que se dedican a otras tareas, principalmente a la ejecución del cuidado. Por otra parte, si bien la investigación mostró una relación de débil significación entre el número de funcionarios de las DD y el número de funcionarios por NNA en el desempeño en planificación, surgen hallazgos no concordantes entre lo que las DD reportaron respecto al grado de cumplimiento en planificación que estuvo en el entorno del $85 \%$, y lo manifestado por las instituciones externas acerca de la escasa planificación a nivel de las acciones que deberían cumplirse a nivel territorial. 
Gráfico 1. Desempeño en Planificación, Atención y Supervisión de las DD

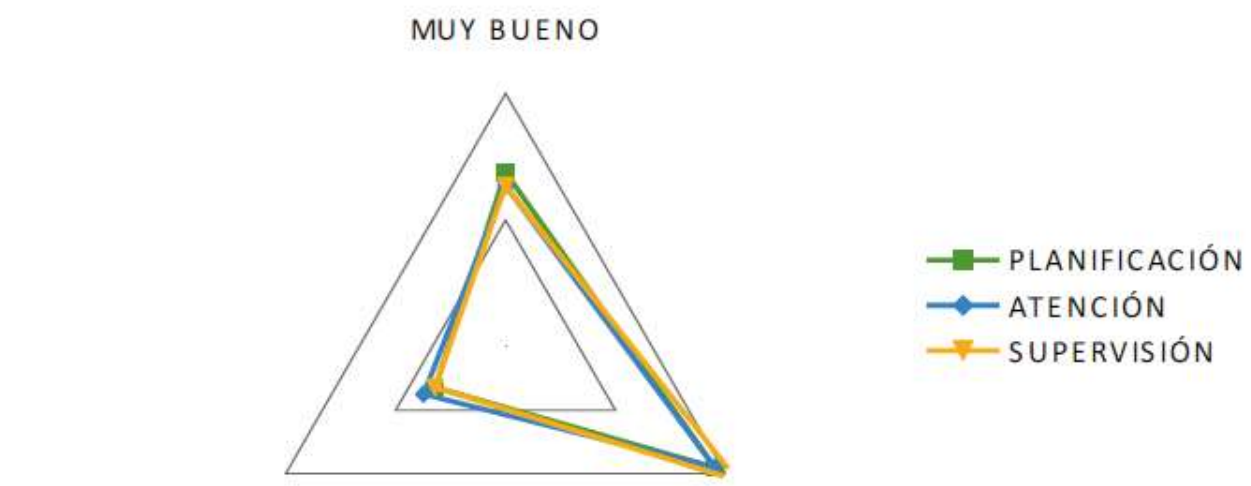

Respecto a la ejecución de cuidado en territorio, la investigación mostró que cerca del 95\% de las DD catalogan la atención en territorio como buena-muy buena y un $5 \%$ como insuficiente, contando todas ellas en su estructura con RRHH dedicados a la misma y con un reporte del $90 \%$ de las DD que dedica RRHH exclusivamente al cuidado. En este aspecto, si bien las direcciones ven ejercer sobre su capacidad de gestión un nivel de demanda comunitaria natural por las condiciones socioeconómicas de las familias o por los vínculos sociales, se encuentran con una presión muchas veces incontrolable de las instituciones que solicitan respuestas, principalmente frente a la posición del Poder Judicial que determina internación. Frente a esta situación del cuidado INAU asume la responsabilidad al extremo de tener que dar respuestas que, si bien involucran a NNA, éstas no son de competencia ajustadas al organismo y a punto tal que en muchas de las solicitudes de respuesta se identifica protección con internación produciéndose por ello la vulneración de las situaciones de NNA y sus familias y en la que INAU responde para no incurrir en desacato.

Si bien esta demanda de cuidado es percibida como causante de las dificultades de desempeños en planificación y rectoría, la investigación mostró que la correlación existente entre el número de funcionarios de las DD y el desempeño en las áreas es muy débil y no significativa. Sin embargo, con respecto a la gestión de la planificación la institución se encuentra en un proceso de readecuación de su gestión y catalogan a la misma como en una etapa muy germinal, pero considerando la incorporación de actores multidisciplinarios a los equipos de dirección de las DD. En esto hay coincidencia con lo expresado por las instituciones externas que plantearon que la amplia gama de respuestas inhibe de recursos y energías en la gestión para las actividades que realmente la institución debería 
desarrollar. Esto además es alimentado por la cultura institucional, y ciertas resistencias a los cambios de los trabajadores y de las propias instituciones contratadas.

En relación a la rectoría, la investigación en las DD mostró que todas cuentan con funcionarios para desarrollarla, aunque solo el $10 \%$ de ellas cuenta con RRHH exclusivos para la misma, siendo el porcentaje de cumplimiento en el entorno del 80\%. Ésta se ejerce específicamente sobre los proyectos de ejecución y de gestión en el sistema INAU en forma directa e indirecta a través de los convenios existentes y con aparente dependencia de los liderazgos locales. Por otra parte, en referencia a la eficiencia de la rectoría no se encontró que la misma tuviera una dependencia significativa en relación a la cantidad de RRHH o número de funcionarios de la institución por NNA de la DD. Si bien el área programática de la mesogestión institucional coordina acciones vinculadas la rectoría, ésta no se desarrolla en la interinstitucionalidad debido a que INAU carece de capacidad y condición jurídica para poder ejercerla. Otro aspecto que surgió en referencia a la rectoría también es la tensión que surge como consecuencia del conflicto que se genera ante la situación en la que la institución se controla a sí misma por asumir los roles de ejecución y supervisión en sí misma, además de que 129 no se cuenta con quien pueda evaluar su gestión, su ejecución de gastos y acciones.

En referencia a desempeño vinculado al cumplimiento de actividades, la correlación de Spearman aplicada a las variables de porcentaje de cumplimiento en las áreas de planificación y supervisión contra el desempeño de las mismas, mostró una asociación débil y no significativa con un coeficiente de 0.307 para el área de planificación y un valor de $\mathrm{p}=0.201$, mientras que para el área de supervisión los valores fueron Coef $=0.095$ y $\mathrm{p}=0.698$ respectivamente. (Tabla $5)$.

La investigación mostró además que las DD evalúan un alto porcentaje de cumplimiento de la gestión, con valores de asociación significativa. Pero la misma es desarrollada principalmente en INAU e instituciones contratadas, centrándose principalmente a estos proyectos sin abarcar acciones en PP de NNA. En este

Tabla 5.

Desempeño en Planificación y Supervisión de las DD.

Desempeño

Supervisión

Desempeño $\quad$ p $\quad$ Coef

Planificación $\quad 0.00^{* * *} \quad 0.899++$

Nota: El Valor de p se calculó mediante la prueba Tau-b de Kendall 
sentido existe coincidencia en lo hallado en las entrevistas, en el entendido de que la institución no posee una estructura de poder propia debido al lugar jerárquico en la que se ubica, y que esto la limita frente a agencias de mayor jerarquía como los ministerios. Sin embargo, la participación desde la interinstitucionalidad le permite generar parte de liderazgo en el escenario nacional de PP de NNA como por ejemplo el Consejo Nacional Consultivo de los Derechos del Niño y el Adolescente. Este análisis del lugar jerárquico y las relaciones de incidencia que posee el INAU como rector de PP de NNA sobre otras instituciones limitan su rol a la vez que la podrían haber inducido a una gestión hacia adentro, en la que se asumen roles de institución total, y que parecerían responder a una característica que históricamente ha formado parte de la institución. Este aspecto, que no surgió de la recolección de datos a la interna de la institución sino que fue una visión externa que surgió a partir del grupo focal, muchas veces juega en articulación con las instituciones de la sociedad civil, y puede estar alimentada por lo sola que se encuentra la institución para dar las respuestas territoriales que se presentan y que habría que evaluar si es correcto que la institución tenga que asumir todos los roles que lleva a cabo, máxime con el lugar que ocupa y la jerarquía que posee.

\section{Conclusiones}

La presente investigación partió de una hipótesis planteando que los preceptos institucionales y lineamientos estratégicos del INAU definidos por las áreas de mesogestión, son eficaces cuando logran una aplicación plena a nivel de la microgestión en el rol ejecutor-territorial, pero también en los roles programático y fiscalizador. Para verificar la hipótesis, se procedió a realizar un estudio no experimental, de carácter exploratorio y descriptivo, mediante estudio de casos múltiple, recurriendo a procedimientos cuantitativos y cualitativos. Para ello se construyeron indicadores que se aplicaron mediante una encuesta a las 19 DD que relevaron el funcionamiento de los niveles de gestión territorial (microgestión). Además se utilizaron las técnicas de entrevistas en profundidad a representantes de los niveles centrales de mesogestión, de grupo focal a actores extra institucionales con incidencia en infancia y adolescencia y revisión documental. De esta forma se procedió a indagar y analizar en el INAU los lineamientos estratégicos establecidos y los preceptos institucionales buscando profundizar en su conocimiento, entendiendo que son eficaces cuando logran una correcta aplicación a nivel territorial en los roles de planificación, ejecución, pero también fiscalizador de la PP de NNA.

En este sentido, en relación al rol del Estado que expone Oszlak (2011), ese papel en PP de NNA que responde a la coyuntura socio-histórica actual es asumido por una institución a la que se le asignan cometidos a través de la Ley, y que elabora un plan estratégico priorizando áreas de intervención en función de lo mandatado por el legislador y poniendo de manifiesto su rol frente a la sociedad. Estas acciones estratégicas que son incorporadas a la agenda pública devienen en una institución que lleva adelante un plan estratégico para dar respuesta a una situación de gran interés social. De acuerdo a lo que expresa Aguilar Villanueva (2003), esta acción institucional en coordinación con actores del Estado en interinstitucionalidad, de la sociedad civil a través de convenios o participando 
de consejos deliberantes, definen al INAU como actor clave en PP en NNA, constituyéndose así en la agencia estatal para la atención de una situación social que se considera relevante y en la que ha sido necesaria instrumentar acciones de acuerdo a la necesidad de las infancias a lo largo del tiempo. De esta forma el INAU responde en su rol en una forma organizativa que modifica recientemente su estructura y plasma en un documento de readecuación institucional que es base del presente estudio y desde donde establecen diferentes niveles de gestión. El análisis del nivel de microgestión mostró que casi el 11\% de los NNA del país se encuentran vinculados a la institución INAU bajo algún tipo de proyecto. Los resultados cuantitativos arrojaron la mayoría de ellos son a través de ejecución de cuidados en todas sus formas y que para dar cumplimiento a ello la institución dedica una importante dotación de recursos en esta tarea, en promedio casi el 90\% de los RRHH. Estos datos son coincidentes con los que se encontraron en los relevamientos cualitativos tanto de las entrevistas a los niveles de mesogestión como de los actores externos participantes del grupo focal. En este sentido, hay que destacar que existen coincidencias en el importante esfuerzo institucional que se desarrolla para poder dar cumplimiento a las necesidades de territoriales de NNA.

Por otra parte, también se surgió de la investigación que esta redirección de recursos hacia la gestión del cuidado debilita notoriamente el rol de planificación que debe llevar adelante el instituto, pero principalmente el de rectoría. Respecto a ésta última, esta debilidad surge de los resultados del estudio obtenidos de los instrumentos tanto a nivel cuantitativo como cualitativo, pero principalmente de éstos últimos es decir, a partir de las entrevistas del nivel meso y a través del análisis que las instituciones externas realizaron. En este sentido hay que destacar las diferencias entre lo reportado por las $\mathrm{DD}$, que evalúan en buena medida la rectoría a nivel territorial, pero la misma se desarrolla casi exclusivamente sobre las instituciones contratadas por INAU para el desarrollo de cuidados y no en una visión más amplia de acuerdo a lo que plantean los cometidos institucionales. Si bien se encontraron valores de asociación significativa a nivel cuantitativo sobre cumplimiento, los resultados no son coincidentes con lo hallado a nivel cualitativo dado que mostraron un nivel de rectoría que no se encuentra alineado a los mencionados establecidos por ley. Es importante señalar la necesidad de haber llevado adelante un análisis de la gestión de la institución considerando los ejes plan de acción, la ejecución del plan y la rectoría, tal como lo manifiesta Ospina (2002) y Bryson (1998), quienes reseñan la necesidad de someter a evaluación los resultados de la Administración Pública. El nivel meso que ha sido estudiado en esta investigación responde a los mandatos macros (definidos a nivel político), que establecen la PP de NNA y define una estrategia de trabajo que está a cargo de las SDGP y SDGT. Estas áreas coordinan en forma horizontal desde cada especialidad su gestión, pero de forma unificada a nivel nacional, definiendo preceptos institucionales que son recogidos por el nivel micro, representado en las DD que son la expresión del rol de INAU a nivel territorial. La investigación mostró que, si bien se reciben lineamientos uniformes, las DD los reproducen con ciertas adaptabilidades de gestión que obedecen a las demandas locales y que el nivel meso ha identificado y, en base a ello ha iniciado un proceso de unificación de gestión y 
respuesta. Cuando se ahonda en el análisis, existen coincidencias en los resultados obtenidos en los que la acción del cuidado es la mayor respuesta brindada, pero demanda gran cantidad de esfuerzo institucional.

Respecto al rol fiscalizador, si bien la institución tiene asignado el rol rector de la PP de infancia, se encontraron resultados coincidentes que muestran que la misma se aplica a las propias instituciones que trabajan para INAU y que existe escasa incidencia a nivel de la PP nacional dado el lugar jerárquico que ocupa en el escenario estatal, aunque algunas experiencias de integración interinstitucional han permitido mejor posicionar el accionar del instituto en este rol. Cuando retomamos la hipótesis que se plantea en la investigación y su construcción a través de las preguntas principal y subsidiarias, podemos considerar que la eficacia en términos de desempeño a nivel de los niveles meso y micro gestión de INAU se caracterizan por tener identificados los roles atribuidos, a decir de Oszlak y O \Donnell, (1976), asumen la resolución de los problemas instalados en la agenda social de NNA, temas que están socialmente problematizados y que son priorizados en función no solamente de su volumen sino también y principalmente del valor social que poseen. Sin embargo, cuando profundizamos en las características de esos roles atribuidos y asumidos, se pueden describir algunos ejes de debate o discusión que se derivan de los resultados obtenidos y que se dan tanto a nivel macro como de meso y micro gestión institucional, aunque el primero no es motivo del presente estudio:

Primer eje de discusión: el rol institucional asignado a INAU y su lugar jerárquico en las PP de NNA.
A partir de la documentación vigente, la legislación asigna un cometido al INAU orientado a darle un lugar a la infancia y priorizarla en el proyecto de las PP que éste plasma en un plan estratégico de PP de NNA. Sin embargo, surge de la investigación que ese rol no puede ser ejercido plenamente debido al lugar jerárquico que el instituto ocupa a nivel interinstitucional relacionándose con el Poder Ejecutivo a través de MIDES, y poniéndose de manifiesto desde las capacidades limitadas de convocatoria e incidencia de la institución hasta en el escaso o nulo poder de veto que posee en temas que involucran a NNA cuya dirección política es ejercida por los Ministerios con competencia en el tema. Por otra parte, se identificaron experiencias de liderazgo en temas específicos como los programas SIPIAV y CONAPEES, que fueron evaluados como muy positivos y con articulación satisfactoria, y también se debe mencionar el papel de liderazgo que el INAU lleva adelante a través de la Comisión de Primera Infancia, Infancia y Adolescencia en el marco del Consejo Nacional de Políticas Sociales, y que no solamente la colidera en conjunto con MIDES, sino que de ese espacio surgió la Estrategia Nacional para la Infancia y la Adolescencia 2010-2030.

Segundo eje de discusión: los vínculos en la gestión de la PP de NNA.

Probablemente se desprenda del punto anterior, como consecuencia del nivel de jerarquía de INAU en el escenario estatal de PP de NNA. Un aspecto que se destacó a nivel de territorio es la gama de propuestas de PP de diferentes organismos estatales como ser intendencias y alcaldías que poseen no solamente fortalezas a nivel local, sino que también reciben recursos desde el gobierno central y que en muchos casos 
están ausentes de la política pública de infancia, porque elaboran su propia política departamental independientemente del quehacer del INAU. Esta característica también se percibió a nivel macro, ya explicitado en el punto anterior. Sin embargo, se destaca el papel del Consejo $\mathrm{Na}$ cional Consultivo de los Derechos del Niño y el Adolescente, el cual fue evaluado como un espacio de muy buena articulación interinstitucional en la gestión de PP de infancia y no solamente a nivel estatal sino también en articulación con la sociedad civil.

Tercer eje de discusión: las dificultades de ejecución de los preceptos institucionales.

Si bien parece estar definido a nivel político y de gestión meso institucional los lineamientos y acciones a llevar a cabo en PP de NNA en los aspectos programático, ejecutor y fiscalizador, la investigación puso de manifiesto las importantes dificultades para llevar adelante la programación, pero principalmente la rectoría. Y esto en cierta medida parecería ser resultado de la alta demanda comunitaria, consecuencia de las condiciones socioeconómicas de las familias o por vínculos sociales, pero también debido a la demanda de instituciones que solicitan respuestas a INAU, principalmente del Poder Judicial, que en varias ocasiones determina internación como medida de protección hacia NNA y en la que la institución no parece poseer socios para dar dichas respuestas.

Cuarto eje de discusión: los alcances del concepto de rectoría.

Como parte del punto anterior, la investigación puso en evidencia varios elementos en referencia a este aspecto del rol institucional. Por un lado, a nivel micro las DD reconocen la rectoría como aspecto importante de la gestión y que es llevada adelante con buenos niveles de desempeño, pero al analizar la misma, y su alcance, su aplicación abarca a las instituciones de cuidado contratadas por INAU, y no considera niveles más amplios en línea con el rol institucional. Este aspecto también se puso en evidencia en la investigación a nivel cualitativo, en donde queda en evidencia la necesidad de la institución de marcar liderazgo, delimitar, responsabilidades, ejercer la supervisión y contralor a nivel de todo el país y en todas las áreas, y que se visualiza a nivel meso, pero no tiene correspondencia a nivel territorial.

Quinto eje de discusión. El ejercicio de los diferentes roles en una misma institución.

Al respecto, surge la situación de posible debate, ya presentado por otros autores, en referencia al posible conflicto que genera que la misma institución que lleva adelante el rol de cuidado ejecute también el rol programador del cuidado, pero principalmente el rol de ejercer la rectoría sobre sí misma. Si bien existen algunas experiencias a nivel nacional de separación de la gestión ejecutora territorial de la planificadora-rectora en otras áreas del Estado y decididas a nivel político (como en el caso de ASSE, por ejemplo), y que han tenido como consecuencia un mejor desarrollo de la gestión asistencial pero también en los niveles de planificación de políticas de salud, principalmente en el rol fiscalizador a cargo del Ministerio de Salud Pública. Cabría preguntarse si, independientemente del conflicto "de controlarse a sí mismo", no se lograrían mejoras en niveles de atención, de planificación, pero principalmente en la rectoría de PP de NNA si esta gestión se desarrollara en una institución separada de la que ejecuta el cuidado y ubicada en un nivel jerárquico superior. 
Sexto eje de discusión: los niveles de uniformidad de funcionamiento de las DD.

Dado que la gestión pública supone un diseño institucional que debe producir cierta coherencia en las prácticas de los distintos niveles de la administración y más allá de las respuestas de los niveles territoriales, las DD deben responder a ello pero también adaptarse a las demandas locales específicas. El instituto ha identificado así la necesidad de uniformizar los criterios de gestión territorial, generando procesos orientadores que se iniciaron con la conformación de equipos de dirección multidisciplinarios que integran las DD y que se estima permitirán una gestión más integrada y no tan centrada en liderazgos individuales.

\section{Comentarios Finales}

De acuerdo a la presente investigación, se puede concluir que la institución INAU se encuentra en un proceso de readecuación caracterizado por un cambio de modelo de gestión centrado en el NNA y su derecho a vivir en familia y en el cual existen líneas estratégicas comunes desde el nivel central de mesogestión hacia el territorio. Este nuevo modelo es germinal, se encuentra sometido a la presión de la cultura institucional y ha iniciado un proceso incorporado equipos de conducción multidisciplinarios a las DD buscando la uniformidad de la respuesta en la gestión con adaptabilidad local. Los preceptos institucionales arrojaron variados niveles de desempeño y cumplimiento a nivel de la microgestión. Se destaca principalmente en el área de ejecución de cuidado que también responde al gran nivel de demanda de atención existente y en el que el INAU parece ser el único con capacidad de respuesta siendo la institución que asume este aspecto en NNA. Esto compromete en cierta medida las otras dos áreas de gestión, principalmente la supervisión. En referencia a este último rol, la rectoría se aplica principalmente en instituciones contratadas por el instituto y esto podría también ser consecuencia del nivel jerárquico que ocupa el INAU en el escenario nacional de la PP de NNA. Sin embargo, hay experiencias de la participación del instituto en espacios de interinstitucionalidad en los que el rol rector es evaluado satisfactoriamente. El estudio de casos con variadas metodologías permitió poner de manifiesto la existencia de prácticas con un alto compromiso institucional. Sin embargo, esta afirmación no implica desconocer ciertas limitaciones y conflictos que la institución posee en el mapa estatal de PP de NNA de acuerdo al rol otorgado, requiriendo un estudio más en profundidad acerca de cómo se desarrollan la PP de infancia, centrado en la protección integral de NNA, su derecho una vida libre de violencias y a vivir en familia. 


\section{Referencias Bibliográficas}

Aguilar Villanueva, L. (1996). La implementación de las Políticas. México. Miguel Ángel Porrúa.

Aries, P. (1987). El niño y la vida familiar en el antiguo régimen. Taurus.

Cerutti, A., Canetti, A., Duarte, D. y Parafita, D. (2014). Políticas Sociales para la Infancia en el Uruguay con Énfasis en las Edades Tempranas. Propuestas de Monitoreo del Bienestar infantil. Centro Interdisciplinario de Infancia y Pobreza, Espacio Interdisciplinario - Udelar.

Morlachetti, A. (2013). Sistemas nacionales de protección integral de la infancia: fundamentos juridicos y estado de aplicación en América Latina y el Caribe. CEPAL.

Monge, E. (2010). El Estudio de Casos como Metodología de Investigación y su importancia en la dirección y Administración de Empresas. Revista Nacional de Administración, 1(2), 31-54.

Neiman, G. y Quaranta, G. (2006). Los estudios de casos en la investigación sociológica. En: I. Vasilachis (2006). (Coord.), Estrategias de investigación cualitativa. Gedisa.

Ospina, S. (2002). Evaluación de la gestión pública: conceptos y aplicaciones en el caso latinoamericano. En V Congreso Internacional del CLAD sobre la Reforma del Estado y de la Administración Pública, Santo Domingo, Rep. Dominicana: 24-27 octubre de 2000. Oszlak, O. (2011). El Rol Del Estado: Micro, Meso, Macro. En VI Congreso de Administración Pública. Asociación Argentina de
Estudios de Administración Pública y la Asociación de Administradores Gubernamentales, Resistencia, Chaco.

Teddlie, C. y Tashakkori, A. (2003). Major Issues and Controversies in the use of Mixed Methods in The Social and Behavioral Sciences. Sage.

Yin, R. (1994). Case Study Research: Design and Methods. Sage.

\section{Documentos Consultados}

Consejo Nacional de Políticas Sociales (2008). Estrategia Nacional para la Infancia y la Adolescencia. 2010-2030. http:// www.inau.gub.uy/institucional/documentos-institucionales.

Gobierno Provisorio de la República Oriental del Uruguay. Código Civil para el Estado Oriental del Uruguay. 23 de enero de 1868. https://archive.org/details/CodigoCivilParaElEstadoOrientalDe/page/ n35/mode/2up

Fondo de las Naciones Unidas para la Infancia (1989). Convención de los Derechos del Niño. https://www.bibliotecaunicef.uy/doc_num.php?explnum_id=146

Instituto del Niño y Adolescente del Uruguay (2019). Datos de Población y Proyectos de INAU. Diciembre de 2019. https:// catalogodatos.gub.uy/dataset/inau-poblacion_y_proyectos 
Instituto del Niño y Adolescente del Uruguay (2019). Estudio de Población y Proyectos de INAU diciembre de 2019. https://www.inau.gub.uy/estudios-de-poblacion-y-proyectos/item/2184-ano-2019

Instituto del Niño y Adolescente del Uruguay (2017). INAU en Cifras $\bigotimes$ Año: 2017. http://www.inau.gub.uy/inau-en-cifras/ item/1933-inau-en-cifras-ano-2017_1933 Instituto del Niño y Adolescente del Uruguay (2018). Manual de Estructura y Funciones. https://www.inau.gub.uy/institucional/normativa /item/29-manual-defunciones.
Instituto del Niño y Adolescente del Uruguay (2019). Metadatos de Población y Proyectos de INAU. Diciembre de 2019. https://catalogodatos.gub.uy/dataset/inau-poblacion_y_proyectos

Instituto del Niño y Adolescente del Uruguay (2019). Plataforma Integrada de Transparencia y Análisis para la Gestión de la Administración. http://www.inau.gub. uy/pitanga-panel-de-proyectos

Instituto del Niño y Adolescente del Uruguay (2015). Readecuación organizacional del INAU -21 de diciembre de 2015. https://www.inau.gub.uy/llamados/llamados-aconcurso/download/3936/1636/16 [consulta: 15 jul 2020]. 